\title{
Lattice dynamics of methane and xenon hydrate: Observation of symmetry-avoided crossing by experiment and theory
}

\author{
J. Baumert, ${ }^{1}$ C. Gutt, ${ }^{2}$ V. P. Shpakov,${ }^{3,4}$ J. S. Tse, ${ }^{4}$ M. Krisch, ${ }^{5}$ M. Müller, ${ }^{1}$ H. Requardt, ${ }^{5}$ D. D. Klug, ${ }^{4}$ \\ S. Janssen, ${ }^{6}$ and W. Press ${ }^{7}$ \\ ${ }^{1}$ Institut für Experimentelle und Angewandte Physik, Universität Kiel, Germany \\ ${ }^{2}$ Experimentelle Physik I, Universität Dortmund, Germany \\ ${ }^{3}$ Chemistry Department, University of Western Ontario, Ontario, Canada \\ ${ }^{4}$ Steacie Institute for Molecular Science, National Research Council of Canada, Ottawa, Canada \\ ${ }^{5}$ European Synchrotron Radiation Facility, Grenoble, France \\ ${ }^{6}$ Paul Scherrer Institute, Villigen, Switzerland \\ ${ }^{7}$ Institute Laue-Langevin, Grenoble, France
}

(Received 18 August 2003; published 3 November 2003)

\begin{abstract}
The collective dynamics of methane and xenon hydrate in the energy range of $\pm 20 \mathrm{meV}$ and the $\mathrm{Q}$ range of $1.5-11 \mathrm{~nm}^{-1}$ has been investigated by inelastic $\mathrm{x}$-ray scattering and compared to results from inelastic neutron scattering experiments and lattice dynamical calculations. The experiment focused on the low-frequency phonon dispersion curves that were found to point towards the existence of an avoided crossing between the acoustic lattice phonons and the localized guest modes. The calculations reproduce the experimental spectra and show that the localized vibrations of the guest molecules or atoms are mixed with the collective host lattice vibrations, leading to a damping of the intensity of the acoustic host lattice phonons. This observation supports the idea of a resonant scattering mechanism for the strong guest-host phonon interactions in clathrate hydrates.

DOI: 10.1103/PhysRevB.68.174301

PACS number(s): 63.20.Kr, 63.20.Dj, 63.20.Pw, 92.40.Sn
\end{abstract}

\section{INTRODUCTION}

Clathrate hydrates, which are a special class of inclusion compounds, have attracted considerable interest in recent years. The interactions between the guest atoms or molecules and the water network make gas hydrates a model system for the study of hydrophobic interactions, relevant for a fundamental understanding of the water and repulsive water-guest potentials ${ }^{1}$ and in problems of protein folding in biology. ${ }^{2}$ Furthermore, large deposits of natural gas hydrate (e.g., methane hydrate) have been discovered on the ocean floors and in arctic permafrost areas, and are considered as a potential future energy resource, for their possible contribution to the greenhouse effect. ${ }^{3}$ In methane and xenon hydrate the methane molecules or xenon atoms are situated in cages formed by an icelike network of H-bonded water molecules. Both hydrates have been identified as being of cubic structure I of the clathrate family ( $a=11.83 \AA$ ), forming six large ellipsoidal and two small spherical cages in the unit cell. ${ }^{4,5} \mathrm{In}$ the case of methane hydrate each cage is occupied by one guest molecule, whereas in xenon hydrate only $80 \%$ of the small cages are occupied.

As gas hydrates consist of more than $80 \%$ of hydrogenbonded water molecules, many of their physical properties resemble those of ice, more specifically ice $\mathrm{I}_{h}$. This is, however, not true for the thermal conductivity $\kappa$, which is unusually low and displays a temperature dependence similar to that of glasses ${ }^{6,7}$ despite the crystalline character of the gas hydrates. A similar behavior of $\kappa$ has also been found in semiconductor clathrates, ${ }^{8,9}$ in which small metal atoms are encapsulated in cages formed by semiconducting atoms like, e.g., Si or Ge. This anomalous behavior of the thermal conductivity in clathrate compounds is attributed to a resonant scattering of the acoustic phonons by the localized guest vibrations in the cages. In the resonant scattering mode ${ }^{10}$ it is hypothesized that an avoided crossing between acoustic (host)-lattice phonons and localized guest modes of the same symmetry leads to a mixing of guest and host modes with an energy exchange as a consequence. Results of recent inelastic incoherent neutron scattering experiments pointed towards a coupling between guest and host vibrations in the phonon density of states of xenon hydrate ${ }^{11,12}$ as well as in various silicon clathrates. ${ }^{13,14}$

In this paper we present a measurement of the collective dynamics of a clathrate hydrate compound by using very high energy resolution inelastic x-ray scattering (IXS). Comparing these results with results from inelastic neutron scattering (INS) and lattice dynamical calculations, we can present experimental evidence of an avoided crossing between the acoustic lattice phonons and the localized guest branches.

\section{EXPERIMENTAL DETAILS}

Both the methane and the xenon hydrate samples were synthesized at the National Research Council, Canada, in a process described elsewhere. ${ }^{7}$ For the x-ray experiments fully protonated samples were prepared. Additionally a partially deuterated $\mathrm{CH}_{4}-\mathrm{D}_{2} \mathrm{O}$ methane hydrate sample was used for the inelastic neutron scattering experiments. From diffraction experiments it is known that the nominal stoichiometry of these hydrates is $\mathrm{CH}_{4} \cdot 5.75 \mathrm{H}_{2} \mathrm{O}$ and $\mathrm{Xe}$ . $6.05 \mathrm{H}_{2} \mathrm{O} .{ }^{5,15}$ The samples were shipped in a flight-proof liquid $\mathrm{N}_{2}$ dewar, as they destabilize at atmospheric pressure when the temperature is raised above $200 \mathrm{~K}$.

As both methane and xenon hydrates only exist in a polycrystalline form, an unambiguous assignment of the ob- 
served modes is only possible in the first Brillouin zone: due to the selection rules in the one-phonon approximation only the longitudinal modes can be observed in the first Brillouin zone. Momentum transfers beyond the first Brillouin zone will excite several longitudinal and transverse phonons at once, thus giving information on the density of states of these modes. ${ }^{16}$ The use of neutrons to determine the collective excitations is virtually ruled out due to restrictions in Q- $\omega$ space at $\mathrm{Q}$ values within the first Brillouin zone (simultaneous conservation of energy and momentum transfer), and due to the incoherent contribution of the hydrogen atoms to the spectrum. As an $\mathrm{x}$ ray can access the small $\mathrm{Q}$ values needed and provide the necessary energy resolution at the same time, the experiments were performed at the beam line ID28 at the European Synchrotron Radiation Facility in Grenoble. By using the silicon $(11,11,11)$ reflection order for both the monochromator and the analyzer an overall energy resolution [full width at half maximum (FWHM)] of 1.5 $\mathrm{meV}$ at $21.747 \mathrm{keV}$ was achieved. For the experiment methane and xenon hydrate samples with a thickness of 15 and 1 $\mathrm{mm}$, respectively, were prepared and loaded onto the precooled cold finger of a closed-cycle helium cryostat. The purity and quality of the samples was assured prior to the inelastic scans by measuring the static structure factors. The ice $\mathrm{I}_{h}$ contamination of both hydrate samples was less than $2 \%$ and they displayed a very good polycrystallinity without preferred orientations. Inelastic scans were then recorded at $T=100 \mathrm{~K}$ in the energy region of $-20-20 \mathrm{meV}$ with momentum transfers of $1.5 \mathrm{~nm}^{-1}<\mathrm{Q}<11.0 \mathrm{~nm}^{-1}$, at lower momentum transfers the contributions from the incident beam were to important. These momentum transfers were chosen in order to cover and extend the measurements beyond the first Brillouin zone. This should give the possibility to assign the observed phonon modes as well as to compare the results with the density of states obtained from the INS experiments.

The inelastic neutron scattering experiment was performed at the FOCUS cold-neutron time-of-flight spectrometer at the PSI, Switzerland. ${ }^{17}$ For the INS experiment the partially deuterated $\left(\mathrm{CH}_{4}-\mathrm{D}_{2} \mathrm{O}\right)$ powder was mounted into a flat aluminum sample cell of $1 \mathrm{~mm}$ thickness and then cooled to $T=150 \mathrm{~K}$ in a liquid helium cryostat. Inelastic measurements were then performed with an initial neutron wavelength of $\lambda=5.0 \AA$ selected from the $(0,0,2)$ reflection order of a pyrolytic graphite monochromator. With the timefocusing option an energy resolution of $0.1-0.4 \mathrm{meV}$ (FWHM) in the range of neutron energy gain of $0-15 \mathrm{meV}$ was obtained. The signal of the empty aluminum sample cell and a flat background have been subtracted.

\section{EXPERIMENTAL AND THEORETICAL RESULTS}

\section{A. Inelastic x-ray and neutron scattering}

In Fig. 1 a selection of inelastic $\mathrm{x}$-ray spectra of methane hydrate is shown at different momentum transfers between 1.5 and $5.0 \mathrm{~nm}^{-1}$. The elastic line at $E=0 \mathrm{meV}$ can be attributed to small angle scattering and to scattering from residual disorder in the powder, and from the sample container. The spectra display a well defined dispersive mode and, from

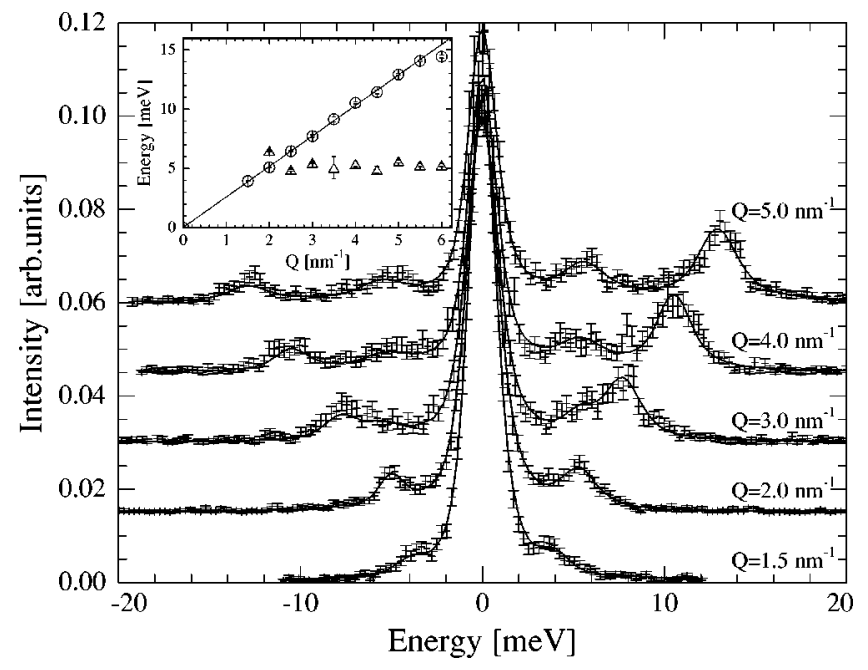

FIG. 1. Inelastic $x$-ray spectra of methane hydrate at several Q values at $T=100 \mathrm{~K}$. The lines are fits to the spectra using Lorentzians convoluted with the experimental resolution. The spectra were normalized to their integrated intensity. The dispersive excitation visible in the spectra is attributed to the longitudinal acoustic lattice mode, whereas the opticlike mode $\left(\Omega_{G}=5.0 \mathrm{meV}\right)$ is attributed to the localized guest vibrations inside the large cage. The inset shows the respective dispersion relation.

$3 \mathrm{~nm}^{-1}$ on, a second nondispersive peak. In order to extract the energy positions $\Omega(Q)$ of the excitations, the spectra were fitted using two Lorentzians, one for each excitation, convoluted with the instrument's resolution. From the fits the energy position of the non-dispersive peak is found to be $\Omega_{G}=5.0 \pm 0.1 \mathrm{meV}$ over the whole observed Q range. The position of the dispersive mode is found to scale linearly with the momentum transfer in the $\mathrm{Q}$ range of $\mathrm{Q}$ $<6.0 \mathrm{~nm}^{-1}$ (inset Fig. 1). This dispersive excitation can be identified with the longitudinal acoustic (LA) host-lattice phonon branch. From the slope of the dispersion of the longitudinal acoustic mode an orientationally averaged sound velocity of $c=3950 \pm 50 \mathrm{~m} / \mathrm{s}$ can be deduced. The value found is very close to that of ice $I_{h}$ and in agreement with very recent results obtained by Brillouin light scattering ${ }^{18}$ and pulse transmission wave speed measurements. ${ }^{19}$

Additional information can be obtained from spectra at higher momentum transfers (Fig. 2). Due to the better contrast - the LA mode moves out of the energy windowadditional broad features can be observed at around 7-10 $\mathrm{meV}$. The broad excitations were fitted with an additional Lorentzian function. On purely statistical grounds, it is found that the energy position of this peak displays a slight dispersion, it ranges from $6.9 \pm 0.2 \mathrm{meV}$ at $\mathrm{Q}=8.0 \mathrm{~nm}^{-1}$ to 9.5 $\pm 0.3 \mathrm{meV}$ at $\mathrm{Q}=11.0 \mathrm{~nm}^{-1}$. As the smallest size of the first Brillouin zone, determined from the $d$ spacing of the first allowed (110) Bragg peak, ${ }^{20}$ is $\mathrm{Q}_{\min }=\pi / d=3.8 \mathrm{~nm}^{-1}$, the spectra in the Q region from 5 to $11 \mathrm{~nm}^{-1}$ are expected to reflect the specific features of the density of states (DOS) of methane hydrate. A comparison of the IXS spectra with INS data can therefore help to attribute the observed modes.

The INS spectrum of the $\mathrm{CH}_{4}-\mathrm{D}_{2} \mathrm{O}$ sample is also shown in Fig. 2. As the host lattice is deuterated the spectrum is 


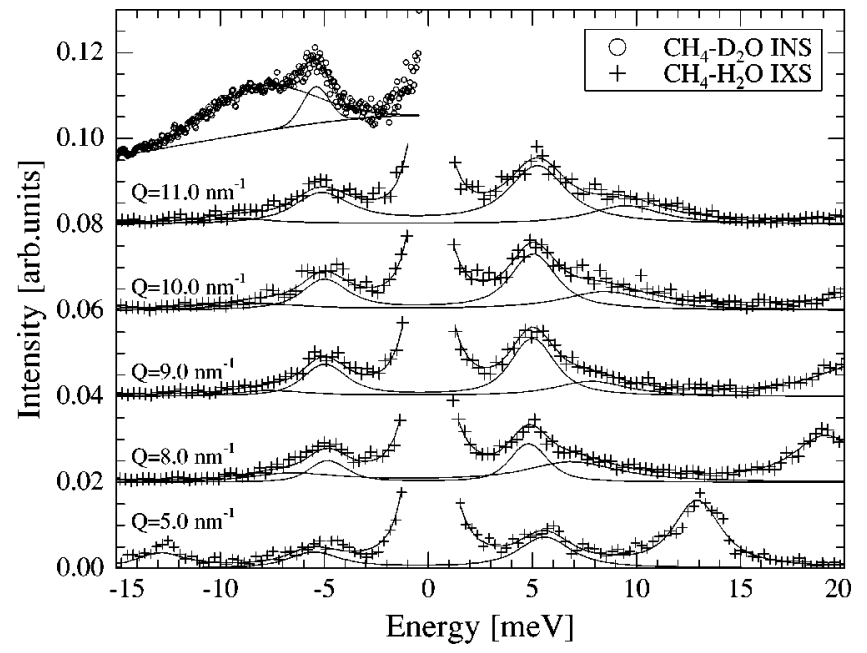

FIG. 2. Inelastic x-ray spectra of methane hydrate at several Q values beyond the first Brillouin zone and an inelastic neutron spectrum of $\mathrm{CH}_{4}-\mathrm{D}_{2} \mathrm{O}$ hydrate are shown. The excitation at $5.4 \mathrm{meV}$ in the INS spectrum and the optic mode at $5 \mathrm{meV}$ in the IXS spectra is assigned to the localized vibrations of methane molecules inside the large cage. The broad shoulder at $8 \mathrm{meV}$ in the INS spectrum arises from both guest and host lattice vibrations. In analogy, the broad slightly dispersive excitation at $7-10 \mathrm{meV}$ in the IXS spectra is attributed to both the TA host lattice phonons and additional guest molecule vibrations.

expected to mostly reflect the dynamics of the methane guest molecules. In the INS spectrum a peak at $5.4 \mathrm{meV}$ with a broad shoulder centered at $8 \mathrm{meV}$ can be observed. The broad background was fitted with a single Lorentzian function, reflecting the rotational excitations of the methane molecules. These molecular rotations are not visible in the IXS experiments, as they are not sensitive for single particle dynamics. From previous INS experiments on several hydrates it is known that the maximum of the transverse acoustic modes is found at about $7 \mathrm{meV}^{21}$ The open cage structure also leads to a "fold-back" of the transverse acoustic (TA) modes, its maximum is located at about $10 \mathrm{meV}$ in the experimental density of states. The energy region below the maxima of the host lattice was found to exhibit the translational vibrations of the guest molecules inside the water cages. ${ }^{12}$ The peak at $5.4 \mathrm{meV}$ in the INS spectrum can therefore be attributed to the methane molecule vibrations inside the large cage, the broad shoulder is assumed to contain contributions from both the additional guest vibrations and the maxima of the TA host lattice modes, which are found at 7.0 and $10.5 \mathrm{meV}$ for $\mathrm{CH}_{4}-\mathrm{H}_{2} \mathrm{O}$ hydrate. ${ }^{22}$

In the IXS spectra the peak at $5 \mathrm{meV}$ appears at $\mathrm{Q}$ $\simeq 3 \mathrm{~nm}^{-1}$, which is close to the smallest size of the Brillouin zone in structure type-I clathrate $\left(Q_{\min }=3.8 \mathrm{~nm}^{-1}\right)$. It might be attributed to the transverse acoustic lattice modes near the zone edge, in analogy to findings in ice $I_{h} \cdot{ }^{23}$ But, contrary to our results, the peak assigned to the transverse modes in ice $I_{h}$ only appears at the zone boundary on increasing $\mathrm{Q}$ and its intensity increases significantly with increasing $\mathrm{Q}$ and quickly dominates the experimental spectra. This intensity behavior indicates a strong transverse symmetry. However, in the IXS spectra of methane hydrate the in- tensity of the peak at $5 \mathrm{meV}$ does not show a strong increase in intensity with increasing wave vector transfer. Additionally, the energy position of $5 \mathrm{meV}$ for the first maximum of the TA lattice modes would disagree with the DOS of the host lattice as determined from INS experiments. We therefore conclude, that it is unlikely that the peak at $5 \mathrm{meV}$ is solely linked to transverse acoustic lattice modes. The resemblance of the IXS spectra at higher Q values with the INS spectrum of $\mathrm{CH}_{4}-\mathrm{D}_{2} \mathrm{O}$ is pointing to a different explanation of the observed spectral features: The excitation at $5 \mathrm{meV}$, in analogy to the interpretation of the INS spectrum, is attributed to the guest vibrations inside the large cage. These vibrations become visible in the spectrum after the intersection with the LA lattice mode at $\mathrm{Q} \simeq 2.5 \mathrm{~nm}^{-1}$, which is still within the first Brillouin zone. This behavior is pointing towards a coupling between the localized guest vibrations and the acoustic host lattice modes. The shoulder at 7-10 meV, which becomes visible at $\mathrm{Q}$ values beyond the first Brillouin zone, cannot be assigned unambiguously. As this excitation does not show a strong increase in intensity with increasing Q though it appears in the same energy region as the first maxima of the TA host lattice modes, it is assumed to contain both contributions from the additional guest vibrations and the maxima of the TA host lattice modes.

Further evidence for our assignment of the non-dispersive mode is provided by IXS spectra of xenon hydrate. The guest and host vibrational density of states of xenon hydrate has already been studied and a coupling of guest and host modes was confirmed. ${ }^{12}$ In Fig. 3 a selection of IXS spectra of xenon hydrate is shown for $Q$ values between 2 and $11 \mathrm{~nm}^{-1}$. Over this whole $\mathrm{Q}$ range the spectra show a broad nondispersive inelastic excitation near the elastic line. The energy position of this feature was determined by fitting it with a Lorentzian function, convoluted with the instrument's resolution. The energy positions range between 2.2 and $3.1 \mathrm{meV}$, with an average of $\Omega_{G}=2.5 \pm 0.3 \mathrm{meV}$. Additionally, at several $\mathrm{Q}$ values below $5 \mathrm{~nm}^{-1}$ a small dispersing feature is visible. The statistics did not allow for a consistent treatment, only at 2.0 and $3.0 \mathrm{~nm}^{-1}$ was a determination of the energy position possible. At these two $\mathrm{Q}$ values the energy positions of the excitation were found to be 5.0 \pm 0.1 and 9.1 $\pm 0.2 \mathrm{meV}$, respectively. These values correspond roughly to the values of the LA mode in methane hydrate, the dispersive feature is therefore assigned to the LA host lattice modes in xenon hydrate. Due to the few data points available we could not determine a reliable velocity of sound for xenon hydrate. The opticlike excitation at $2.5 \mathrm{meV}$ is already observable at the lowest $\mathrm{Q}$ values, and therefore has longitudinal contributions. It can be assigned to the localized guest vibrations with the additional information from INS results on the dynamics of xenon hydrate. From these it is known that the localized guest vibrations of the xenon atoms inside the water cages are found at 2.1, 2.9, and $3.9 \mathrm{meV} .{ }^{12}$ Considering the resolution of $1.5 \mathrm{meV}$ (FWHM) in the present IXS experiment, it can be understood that these vibrations were not resolvable. Instead, a single broad excitation is observed in the spectra, whose energy position matches well with the average of the INS findings. 


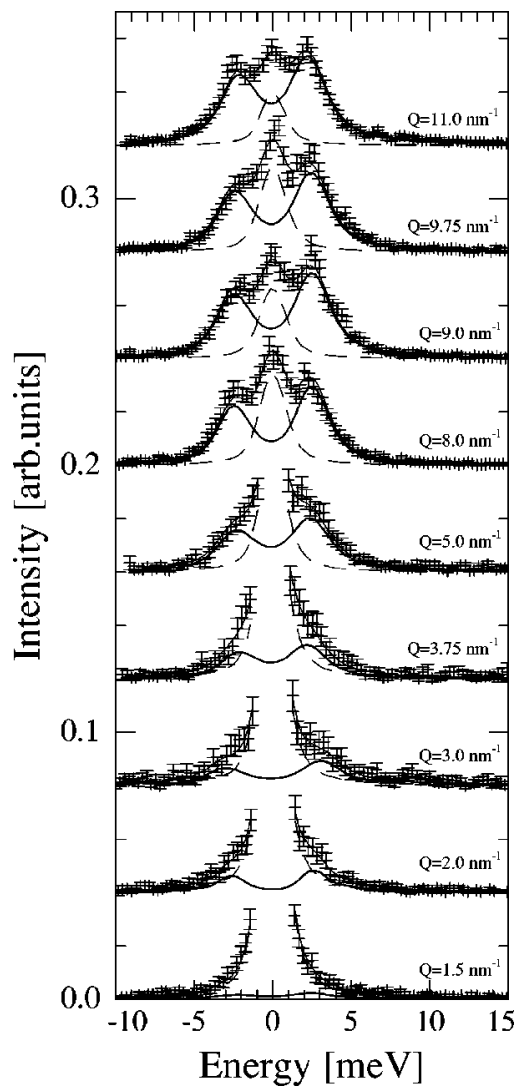

FIG. 3. Inelastic $\mathrm{x}$-ray spectra of xenon hydrate at several $\mathrm{Q}$ values at $T=100 \mathrm{~K}$. The lines are fits to the spectra using Lorentzians convoluted with the experimental resolution. The spectra were normalized to their integrated intensity. The nondispersive feature visible near the elastic line $\left(\Omega_{G}=2.5 \mathrm{meV}\right)$ is attributed to the localized guest vibrations inside the water cages.

In comparison with methane hydrate, the change in the guest species leads to a shift in the vibrational frequencies that accounts for the difference in guest mass and the change in the guest-host interaction strength. The interpretation of the IXS spectra in terms of localized guest vibrations therefore leads to a consistent explanation for both xenon and methane hydrate.

\section{B. Lattice dynamical calculations}

To further validate the correctness of our interpretations and to understand the origin of the observed features, the dispersion relation and the IXS intensities were calculated using lattice dynamics (LD) simulations. The LD calculations were performed with periodic boundary conditions on one proton-disordered unit cell of structure I clathrate hydrate containing 46 water molecules and eight methane molecules or xenon atoms with a lattice parameter $a$ $=11.83 \AA$. The interactions between the water molecules were described by a slightly modified TIP4P potential. ${ }^{24}$ The methane molecules were assumed to be spherical symmetric Lennard-Jones particles ${ }^{25}$ and the OPLS potential was used in the calculations. Therefore the rotations of the methane molecules are not taken into account in the calculation, which is a reasonable simplification as single particle dy-

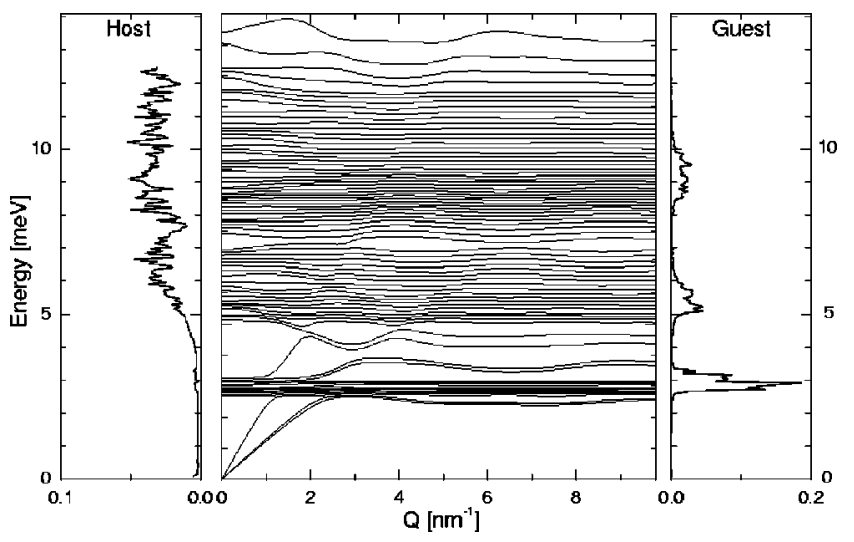

FIG. 4. Calculated powder averaged phonon dispersion of methane hydrate as deduced from 239 random Q directions. The density of states of the host lattice and the guest molecules are displayed on the left and right sides of the dispersion curve, respectively. The characteristic vibrations of the guest molecules are found at 3, 5, and $9 \mathrm{meV}$. The first maxima in the DOS of the host lattice are found at about 6 and $10 \mathrm{meV}$.

namics are not visible in the IXS spectra. The xenon interactions were also described by a Lennard-Jones type potential. $^{26}$ The short-range interactions between the molecules or atoms of different types were defined by the Lorentz-Berthelot mixing rules. The calculated phonon dispersions were averaged over 239 random directions in the Brillouin zone in order to simulate the powder sample. In Fig. 4 the respective dispersion relation of methane hydrate is shown. The dispersive modes near the zone center are the transverse and longitudinal acoustic lattice modes. The calculated orientationally averaged longitudinal sound velocity is $3800 \mathrm{~m} / \mathrm{s}$, in good agreement with the experimental value. Additionally the density of states for both the guest and the host vibrations is shown. The DOS of the host lattice shows that the maximum of the TA modes is at $6 \mathrm{meV}$ and of its "foldback" at $10 \mathrm{meV}$, which corresponds to the values found in the INS experiments. The dynamical characteristics of the water lattice are thus well reproduced by the TIP4P water potential. In the calculated DOS of the guest molecules three peaks at 3,5 , and $9 \mathrm{meV}$ can be observed. They correspond to the characteristic guest molecule vibrations inside the large cage ( 3 and $5 \mathrm{meV})$ and the small cage $(9 \mathrm{meV})$, respectively. The calculated frequencies of the guest modes are thus shifted towards lower frequencies with respect to the measured frequencies by about $30-40 \%$. This points towards an underestimation of the repulsive methane-water potentials. A strong bending of the LA and TA modes can be observed at the crossing points with the guest branches at $\mathrm{Q} \simeq 1.1$ and $\mathrm{Q} \simeq 2.2 \mathrm{~nm}^{-1}$, respectively. This bending is due to an avoided crossing between the localized methane vibrations and the acoustic phonons of the same symmetry. ${ }^{27}$ More avoided crossings between guest and host phonon branches can be observed in the energy region of 5-12 meV.

The calculated orientational averaged dispersion relation of xenon hydrate is shown in Fig. 5. Below $2 \mathrm{meV}$ the TA and LA phonon branches of the host lattice are visible. The theoretical orientationally averaged longitudinal velocity of sound is found to be $3000 \mathrm{~m} / \mathrm{s}$. This value is in good agree- 


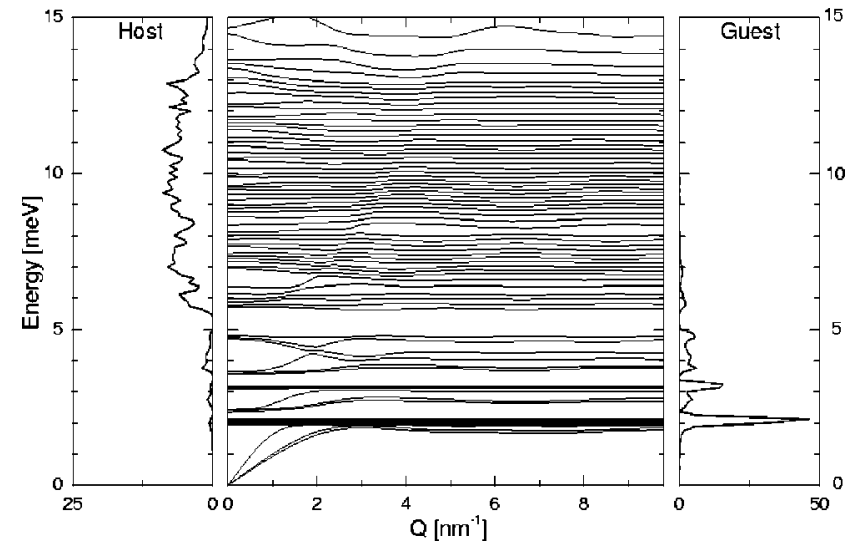

FIG. 5. Calculated powder averaged phonon dispersion of xenon hydrate. The density of states of the host lattice and the guest molecules are displayed on the left and right sides of the dispersion curve, respectively. The characteristic vibrations of the guest molecules are found at about 2,3 , and $4 \mathrm{meV}$. The first maximum in the DOS of the host lattice is found at about $6 \mathrm{meV}$.

ment with experimental results from Brillouin light scattering. ${ }^{28}$ From the calculated DOS it can be seen that the xenon guest vibrations are found at about 2,3 , and $4 \mathrm{meV}$, and correspond to the two different vibrational frequencies inside the large cage and the vibrational frequency inside the small cage, respectively. The values are again in agreement with previous results from INS experiments. ${ }^{12}$ As already observed in the calculated dispersion relation of methane hydrate, the LA and TA modes display a strong bending at the crossing points with the guest branches at 1.2 and $2.4 \mathrm{~nm}^{-1}$, respectively.

In order to compare the calculated dispersion relations with the experimental IXS spectra it is important to take the intensity of the calculated modes into account. The scattering functions $S(\mathbf{Q}, \omega)$ were therefore calculated from the LD results to allow a direct comparison with the experimental results. The scattering functions were also convoluted with a Lorentzian-shaped resolution function, to approximate also the experimental resolution. In a one-phonon approach the scattering function in an IXS experiment can be written as

$$
S(\mathbf{Q}, \omega)=G(\mathbf{Q}, \mathbf{q}, j) \cdot F(\omega, T, \mathbf{q}, j),
$$

where $\mathbf{q}$ denotes the phonon wave vector in branch $j$, $\mathrm{G}(\mathbf{Q}, \mathbf{q}, j)$ is the dynamical structure factor and $\mathrm{F}(\omega, T, \mathbf{q}, j)$ is the response function. ${ }^{16} \mathrm{G}(\mathbf{Q}, \mathbf{q}, j)$ was calculated and averaged for the eigenvectors found in the LD simulations for each of the 239 different directions within the Q range of $0-10 \mathrm{~nm}^{-1}$. The phonon wavevector $\mathbf{q}$ was chosen such that $\mathbf{q}=\mathbf{G}-\mathbf{Q}$, where $\mathbf{G}$ was an appropriate lattice vector for $\mathbf{Q}$ values beyond the first Brillouin zone. Thus, the intensities of both the longitudinal and the transverse modes are calculated. In the harmonic approximation the response function $\mathrm{F}(\omega, T, \mathbf{q}, j)$ of an undamped harmonic oscillator was chosen and on this basis a scattering function $S(\mathbf{Q}, \omega)$ could be calculated.

The resulting theoretical scattering functions for methane and xenon hydrate are shown in Fig. 6. The calculated IXS

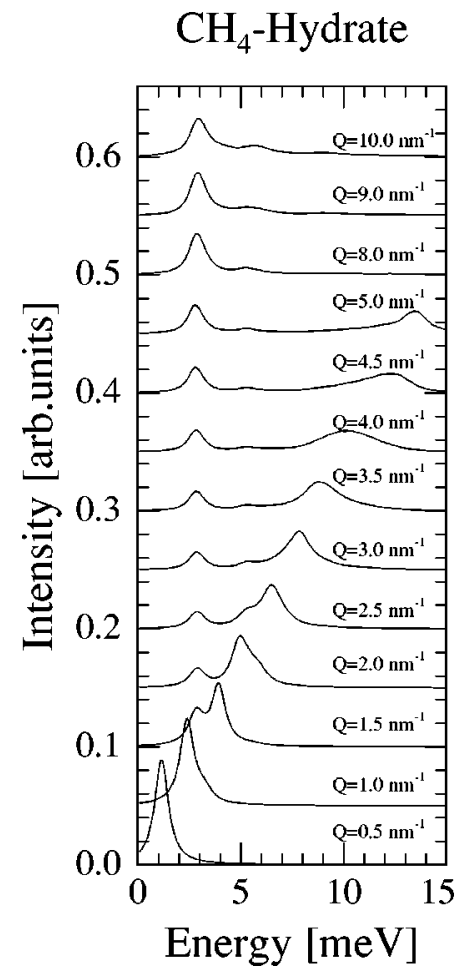

Xe-Hydrate

FIG. 6. Theoretical orientationally averaged IXS scattering function $S(\mathbf{Q}, \omega)$ of methane and xenon hydrate. The calculated spectra are shown for several Q values.

spectra for methane hydrate display two distinct excitations, a dispersive mode, which is the LA host lattice mode, and an optic mode at $\Omega \simeq 3 \mathrm{meV}$, which becomes observable at the crossing with the LA $\left(\mathrm{Q}>1.0 \mathrm{~nm}^{-1}\right)$ mode and corresponds to the methane vibrations inside the large cage. In the case of xenon hydrate the optic guest modes also become observable at the crossing with the acoustic host lattice mode ( $Q$ $>1.0 \mathrm{~nm}^{-1}$ ). In contrast to methane hydrate all of the guest modes are visible in the spectra, though the mode corresponding to the xenon vibrations inside the large cage clearly dominates the spectra. The LA mode, which can be observed throughout the whole $\mathrm{Q}$ range for methane hydrate, loses most of its intensity at the avoided crossing in the case of xenon hydrate, and becomes negligible for $\mathrm{Q}>3.0 \mathrm{~nm}^{-1}$. The characteristic features of the experimental spectra of both methane and xenon hydrate (Figs. 1-3) are thus reproduced by the calculations, which strongly support the interpretation of the spectra in terms of guest modes and host lattice phonons.

\section{DISCUSSION AND CONCLUSION}

A comparison of the experimental and theoretical results for methane and xenon hydrates shows that the spectra strongly depend on the guest species. In the case of methane hydrate a single optic mode, attributed to the guest vibrations, is visible, the LA lattice mode can be observed over a wide $Q$ range, and at higher $Q$ values contributions from the TA lattice modes appear in the spectra. In the experimental and calculated spectra of xenon hydrate all three guest modes 
are observable, although they could not be resolved individually in the experiment due to the instrument's resolution. The LA lattice mode is very weak and becomes quickly negligible, whereas the TA lattice phonons cannot be observed at all. These differences between the spectra of methane and xenon hydrate cannot be solely explained by the difference in scattering cross section. In addition, the difference in the localization of the guest modes (see Figs. 4 and 5) seems to play an important role.

At the zone center, before the crossing of the guest and host modes, the guest molecules or atoms behave like independent Einstein oscillators in the cages, leading to a negligible intensity of the guest modes compared to that of the collective acoustic lattice mode in the IXS spectra. Therefore both methane and xenon hydrate spectra look very similar at low Q values. Especially the calculated spectra at 0.5 and $1.0 \mathrm{~nm}^{-1}$ are almost identical, though they were not accessible in the experiments. It is at the avoided crossing with the LA host lattice branch that the independent localized vibrations of the guest molecules or atoms receive a strong longitudinal polarization through a mixing of the eigenvectors of the guest and host modes. At these $\mathrm{Q}$ values the guest vibrations are no longer independent rattlers in the cages. Instead their vibrations are modulated by the host lattice, leading to a transfer of scattered intensity from the acoustic lattice mode to the guest vibrations and thus to the observed optic modes. The strength of this coupling is dependent on the localization of the guest modes. In the case of xenon hydrate all three guest modes are strongly localized and therefore become observable in the spectra after the avoided crossing. For methane hydrate only the lowest guest mode is localized enough to lead to a visible optic mode in the experimental and calculated spectra. Therefore, the intensity transfer is stronger in the case of xenon hydrate and leads, together with the higher scattering cross section of the xenon atom, to a very weak LA host lattice mode. Phonon relaxation time calculations also predicted a stronger coupling for the TA lattice modes than for the LA lattice modes in xenon hydrate ${ }^{11}$; therefore, the TA lattice phonons are not observed. Methane hydrate shows a weaker coupling and therefore the LA lattice mode is visible over a wide $\mathrm{Q}$ range. The additional guest molecule vibrations and the contributions from the TA host lattice modes are also observable within the broad excitation at about 7-10 meV. The guest-host interaction thus leads to a damping of the intensity of the dispersive lattice phonons at the avoided crossing points in the Brillouin zone.

In conclusion, with the help of IXS, it was possible to determine the collective dynamics of two clathrate hydrates. Combining IXS and INS results, an interpretation of the experimental spectra in terms of acoustic lattice phonons and localized guest vibrations was favored. This explanation of the experimental results was additionally validated by lattice dynamical simulations. The LD calculations reproduced the dynamical properties of the water lattice of both methane and xenon hydrate to a very good degree. Although the frequencies of the guest vibrations were underestimated in the calculations of methane hydrate, they matched the experimental findings for xenon hydrate. The resulting orientationally averaged theoretical scattering functions also reproduce the very specific features of the experimental spectra. From this analysis the existence of an avoided crossing between the acoustic lattice phonons and the localized guest modes can be deduced. It was found to lead to a damping of the intensity of the acoustic lattice phonons, which are closely related to the thermal conductivity of a material. To gain insight into the influence of this avoided crossing on the thermal conductivity an experimental determination of the phonon lifetime in clathrate hydrates would be necessary. This could, in principle, be obtained from inelastic scattering experiments if the phonon peak width could be determined precisely including anharmonic effects in the data analysis.

\section{ACKNOWLEDGMENTS}

This work was partially performed at the Swiss Spallation Neutron Source SINQ, Paul Scherrer Institute, Villigen, Switzerland. This work was funded by the BMBF and DFG within the program GEOTECHNOLOGIEN (Project No. 03GO551A, publication no. GEOTECH-6).
${ }^{1}$ J.S. Loveday, R.J. Nelmes, M. Guthrie, D.D. Klug, and J.S. Tse, Phys. Rev. Lett. 87, 215501 (2001).

${ }^{2}$ M.M. Teeter, Proc. Natl. Acad. Sci. U.S.A. 81, 6014 (1984).

${ }^{3}$ E.D. Sloan, Jr., Clathrate Hydrates of Natural Gases (Dekker, New York, 1998), p. 534.

${ }^{4}$ D.W. Davidson, Y.P. Handa, C.I. Ratcliffe, J.S. Tse, and B.M. Powell, Nature (London) 311, 142 (1984).

${ }^{5}$ C. Gutt, B. Asmussen, W. Press, J.S. Tse, Y.P. Handa, and M.R. Johnson, J. Chem. Phys. 113, 4713 (2000).

${ }^{6}$ R.G. Ross, P. Andersson, and G. Bäckström, Nature (London) 290, 322 (1981).

${ }^{7}$ Y.P. Handa and J.G. Cook, J. Phys. Chem. 91, 6327 (1987).

${ }^{8}$ J.L. Cohn, G.S. Nolas, V. Fessatidis, T.H. Metcalf, and G.A. Slack, Phys. Rev. Lett. 82, 779 (1999).

${ }^{9}$ J.S. Tse, K. Uehara, R. Rousseau, A. Ker, C.I. Ratcliffe, M.A. White, and G. MacKay, Phys. Rev. Lett. 85, 114 (2000).
${ }^{10}$ J.S. Tse and M.A. White, J. Phys. Chem. 92, 5006 (1988).

${ }^{11}$ J.S. Tse, V.P. Shpakov, V.R. Belosludov, F. Trouw, Y.P. Handa, and W. Press, Europhys. Lett. 54, 354 (2001).

${ }^{12}$ C. Gutt, J. Baumert, W. Press, J.S. Tse, and S. Janssen, J. Chem. Phys. 116, 3795 (2002).

${ }^{13}$ P. Melinon, P. Keghelian, A. Perez, B. Champagnon, Y. Guyot, L. Saviot, E. Reny, C. Cros, M. Pouchard, and A.J. Dianoux, Phys. Rev. B 59, 10099 (1999).

${ }^{14}$ E. Reny, A. San-Miguel, Y. Guyot, B. Masenelli, P. Melinon, L. Saviot, S. Yamanaka, B. Champagnon, C. Cros, M. Pouchard, M. Borowski, and A.J. Dianoux, Phys. Rev. B 66, 014532 (2002).

${ }^{15}$ T. Ikeda, S. Mae, O. Yamamuro, T. Matsuo, S. Ikeda, and R.M. Ibberson, J. Phys. Chem. 104, 10623 (2000).

${ }^{16}$ E. Burkel, J. Phys.: Condens. Matter 13, 7627 (2001).

${ }^{17}$ J. Mesot, S. Janssen, L. Holitzner, and R. Hempelmann, J. Neutron Res. 3, 293 (1996). 
${ }^{18}$ H. Shimizu, T. Kumazaki, T. Kume, and S. Sasaki, Phys. Rev. B 65, 212102 (2002).

${ }^{19}$ M.B. Helgerud, W.F. Waite, S.H. Kirby, and A. Nur, in Proceedings of the 10th International Conference on Physics and Chemistry of Ice [Can. J. Phys. 81, 47 (2003)].

${ }^{20}$ F. Hollander and G.A. Jeffrey, J. Chem. Phys. 66, 4699 (1977).

${ }^{21}$ B. Chazallon, H. Itoh, M. Koza, W.F. Kuhs, and H. Schober, Chem. Phys. Phys. Chem. 4, 4809 (2002).

${ }^{22}$ J. Baumert, C. Gutt, M.R. Johnson, W. Press, and J.S. Tse, Institute Laue-Langevin, Annual Report, 2002.

${ }^{23}$ F. Sette, G. Ruocco, M. Krisch, C. Masciovecchio, R. Verbini, and U. Bergmann, Phys. Rev. Lett. 77, 83 (1996).

${ }^{24}$ V.P. Shpakov, P.M. Rodger, J.S. Tse, D.D. Klug, and V.R. Belosludov, Phys. Rev. Lett. 88, 155502 (2002).

${ }^{25}$ O. Forrisdahl, B. Kvamme, and A.D. Haymet, Mol. Phys. 89, 819 (1996).

${ }^{26}$ J.S. Tse, M.L. Klein, and I.R. McDonald, J. Chem. Phys. 78, 2096 (1983).

${ }^{27}$ J.S. Tse, V.P. Shpakov, V.V. Murashov, and V.R. Belosludov, J. Chem. Phys. 107, 9271 (1997).

${ }^{28}$ H. Kiefte, M.J. Clouter, and R.E. Gagnon, J. Phys. Chem. 89, 3103 (1985). 